\title{
Reflets
}

Revue ontaroise d'intervention sociale et communautaire

\section{Intervention auprès d'une adolescente dépressive en milieu scolaire}

\section{Michel-André Beauvolsk}

Volume 7, numéro 1, printemps 2001

Santé mentale et les défis de l’an 2001

URI : https://id.erudit.org/iderudit/026341ar

DOI : https://doi.org/10.7202/026341ar

Aller au sommaire du numéro

Éditeur(s)

Reflets : Revue ontaroise d'intervention sociale et communautaire

ISSN

1203-4576 (imprimé)

1712-8498 (numérique)

Découvrir la revue

Citer cet article

Beauvolsk, M.-A. (2001). Intervention auprès d'une adolescente dépressive en milieu scolaire. Reflets, 7(1), 152-160. https://doi.org/10.7202/026341ar

Tous droits réservés (C) Reflets : Revue ontaroise d'intervention sociale et communautaire, 2001
Ce document est protégé par la loi sur le droit d'auteur. L'utilisation des services d’Érudit (y compris la reproduction) est assujettie à sa politique d'utilisation que vous pouvez consulter en ligne.

https://apropos.erudit.org/fr/usagers/politique-dutilisation/ 


\section{Intervention auprès d'une adolescente dépressive en milieu scolaire}

Michel-André Beauvolsk, Ph.D.

Les mondes scolaire et familial semblent amener parfois des niveaux de stress que les adolescentes ont de la difficulté à gérer. Ajouté à ce phénomène vient celui de la violence à l'école et la quête du sens d'appartenance.

La cliente a été référée par ses parents qui ont identifié des comportements et des attitudes qui les déroutaient. Ils soupçonnaient que leur fille était dépressive et croyaient qu'elle avait tendance à s'isoler trop. Il s'agit d'une jeune femme de 16 ans inscrite en $11^{\mathrm{e}}$ année dans une école secondaire du Nord de l'Ontario. Ses parents lui ont conseillé d'aller en counselling parce qu'ils la trouvaient dépressive, isolée et confuse.

Cet article se veut une illustration d'une intervention utilisant le modèle symbolique expérienciel de Carl Whitaker. Ce modèle utilise les symboles, la musique, le jeu pour faciliter la prise de conscience et supporter le changement voulu par la cliente.

\section{Un très bref survol du modèle de Carl Whitaker}

Né sur une ferme isolée du Nord de l'état de New York, Carl Whitaker a reçu son doctorat en médecine de l'Université de Syracuse en 1936. Il a pratiqué la psychothérapie pendant plus de 40 ans. 
Son approche se situe dans la psychologie expériencielle. Selon sa théorie, la santé est un processus en perpétuel devenir. La famille fonctionne comme un tout formé de trois à quatre générations et est intégrée longitudinalement, c'est-à-dire que la famille et ses sous-groupes et les membres individuels sont reliés à la famille intra-psychique de trois générations et plus. Les symptômes ne sont pas absents de la famille en santé, mais ils deviennent une manière d'accroître l'expérience familiale et, en quelque sorte, sa croissance. Le type de symptômes qu'on y retrouve est habituellement relié aux rôles préétablis pour les membres de la famille et par une fixation de triangles entre les déterminants fonctionnels des deux familles d'origine.

\section{L'évaluation}

L'évaluation dans ce modèle est implicite. Elle débute avec le premier appel téléphonique et se continue lors de la première entrevue. Ici, deux principes opèrent en parallèle. Le premier est relié à notre tentative de faire connaissance, de développer une relation empathique et d'identifier les composantes anxiogènes requises pour pousser la famille à changer. Le deuxième élément est le feu d'un dur labeur. Puisque l'évaluation se veut comportementale et interpersonnelle, il importe aussi de souligner les pathologies culturelles invisibles.

La première entrevue se situe dans une bataille pour la structure. C'est essentiellement à notre demande que le système-client capitule devant les modes d'opération du thérapeute. Il existe aussi en même temps une bataille pour l'initiative. L'initiative de la thérapie doit venir du système-client. Pour Whitaker, un système-client se veut idéalement une famille mais le cas présent concerne un systèmeclient simple, c'est-à-dire une seule personne. Lorsqu'il travaille avec un système-client, Whitaker requiert la présence de toute la famille. Ici, dans le Nord, cela n'est pas toujours possible. Nous présentons quand même la pensée originale de Whitaker et nous appliquons son modèle d'intervention à notre cliente. 


\section{Les buts de l'intervention}

Habituellement, lorsque la famille est présente, les buts de l'intervention de Whitaker consistent à établir un sens d'appartenance parmi les membres de la famille et en même temps d'assurer la liberté individuelle. Pour atteindre ces buts, nous allons :

1) accroitre les stress interpersonnels,

2) faire émerger de la présence familiale un partage de l'anxiété dans un développement d'un nationalisme familial,

3) chercher à accroître les relations familiales avec la famille étendue,

4) pousser pour accroître la relation familiale avec la culture et les membres de la communauté,

5) encourager l'établissement d'un sens des frontières familiales avec une meilleure compréhension et une meilleure connexion avec les attentes de la famille, et

6) faire un effort pour séparer les générations.

Simultanément, avec une structure plus claire, la famille doit apprendre à jouer. Le jeu est universel. Nous faisons un effort pour développer une union entre nous et eux, entre l'équipe thérapeutique et la famille, en utilisant un cycle constant de séparation et de réunion. Nous cherchons à faire éclater le mythe de l'individualité pour que la famille croit en elle même. Il est important que cette croyance soit assaisonnée d'un sens très fort de son absurdité, et chaque membre de la famille doit être plus lui-même, elle-même.

\section{Le rôle de l'intervenant}

Le thérapeute est comme un "entraîneur», «un guide» ou "un grand-père adoptif». Ces rôles demandent une structure, une discipline et de la créativité ainsi qu'une disponibilité personnelle et aidante. 
La première entrevue porte d'habitude sur l'histoire systématique de la famille. Nous essayons alors de connaitre le système émotionnel de la famille en commençant par les membres les plus psychologiquement distants, le plus souvent le père.

Pour joindre la famille, le thérapeute familial doit développer une base empathique avec la famille. Dans le choix des techniques utilisées, nous considérons comme les plus importantes celles qui suivent :

1) redéfinir les symptômes comme des efforts de croissance;

2) modeler des alternatives humoristiques au stress de la vie réelle;

3) séparer le stress interpersonnel et le stress de fantaisie intrapersonnelle;

4) souligner l'importance pour la famille d'éviter de discuter des entrevues entre les sessions;

5) augmenter le désespoir d'un membre de la famille de telle sorte que la famille s'unira autour de lui;

6) confronter effectivement; et

7) traiter les enfants comme des enfants et non comme des pairs. La fin de la thérapie est basée sur une diminution du stress.

Ici,le modèle de thérapie expériencielle de Whitaker est appliqué à une seule personne, ce qui implique que certaines techniques ont été adaptées au besoin.

\section{Première rencontre}

La cliente se présente une fin d'après-midi, vêtue d'une longue robe noire, les lèvres noires et le regard vide. Le contact visuel n'est pas très soutenu et la voix est très faible.

Selon Whitaker, le début de la rencontre se nomme la bataille pour la structure. Il s'agit d'établir une relation de confiance, de collaboration où l'intervenant se définit comme un guide, un entraîneur qui se veut très actif dans le travail de changement que la cliente entrevoit. Whitaker croit qu'un changement chez une personne requiert une amplification de l'énergie dans le système- 
client. Cette première session devient l'occasion de joindre le monde de la cliente en écoutant la narration de sa situation de vie.

Josée, notre cliente, vient d'une famille où le père et la mère occupent des postes administratifs dans de grosses compagnies canadiennes. Elle est la plus jeune de trois enfants. Elle a deux frères, Roland 30 ans et Julien 28 ans. Ses deux frères sont mariés et vivent dans le sud de l'Ontario.

Josée entre dans l'agence et s'assoit dans la salle d'attente. Notre première rencontre commence quelques minutes plus tard. Je lui demande ce qui l'amène. Elle raconte que ses parents l'envoient en counseling. Je lui demande alors ce qu'elle veut. Elle me répond qu'elle ne sait pas trop. Je lui demande alors ce qu'elle ressent d'être ici. Plutôt confuse, me dit-elle d'une voix très basse. Je recadre l'entrevue en affirmant que même si je reconnais que ses parents l'envoient en counseling, elle est la cliente et mon rôle consiste à travailler avec elle sur ce qu'elle veut changer.Je vérifie aussi auprès d'elle si elle se sent bien de travailler avec un intervenant ou si elle préférerait travailler avec une intervenante. Elle me dit qu'elle va essayer cette première rencontre et qu'elle me dira comment elle se sent après cette première session. Nous continuons donc cette session en explorant ensemble ce qu'elle aimerait accomplir et changer. Elle me raconte comment elle se sent seule et inquiète. À l'école, elle doute vraiment de pouvoir réussir sa $11^{\mathrm{e}}$ année. Elle explique comment elle retourne chez elle l'après-midi pour se retrouver seule dans sa chambre et comment elle n'a que peu d'amies. Je lui demande alors de me décrire sa famille. À la maison, elle vit seule avec son père puisque sa mère est très souvent en dehors de la ville en raison du poste important qu'elle occupe dans une compagnie très réputée. Lorsque son père revient du travail, il fait cuire le repas, ils soupent ensemble, puis elle se retrouve seule dans sa chambre. Elle utilise alors Internet ou le téléphone pour rejoindre ses amies et jaser souvent toute la soirée. Elle aime aussi pratiquer la natation où elle s'est méritée plusieurs trophées. Avec ses amies, elle aime aussi jouer au tennis.

Comme la fin de cette session approche, je lui demande alors comment elle se sent et quelle décision elle a prise face au 
counseling. Elle me dit qu'elle va continuer avec moi. Comme le temps est passé très vite, je l'invite alors à amener à la prochaine session, deux mémentos, objets ou souvenirs très significatifs pour elle, qui refléteraient les moments de sa vie où elle était très heureuse.

\section{Deuxième rencontre : les deux mémentos}

Josée arrive à la deuxième rencontre encore un peu perplexe sur ce qu'elle accomplira dans cette session. Elle porte toujours la même longue robe noire. Son regard se fixe encore sur le plancher et elle parle très peu. Je lui demande comment elle va. La réponse se veut presque inaudible. Je lui demande de répéter un peu plus fort. Elle me dit que tout est pareil. Comment ça pareil? Toujours la même routine. Elle raconte comment à la maison elle se sent toujours seule et comment son père ne lui parle presque jamais. Elle passe son temps dans sa chambre à faire ses devoirs, à parler à ses amies au téléphone et à passer beaucoup de temps sur Internet ou à regarder la télévision. Elle se sent vide et sans intérêt.

Nous en venons à ses deux mémentos. Elle a apporté un disque compact de musique de son groupe préféré, Emenem. Nous écoutons trois plages de musique où je l'invite à partager avec moi ce qu'elle aime particulièrement dans ces chansons. Je lui rappelle que les titres des chansons sont plutôt négatifs et déprimants. Elle partage que ces chansons la stimulent et l'invitent à redécouvrir ce qu'il y a de positif dans la vie. C'est paradoxal.

Le deuxième mémento est un cadeau qu'elle a reçu de son amie qui est allée en voyage à Rome et qui a fait le pèlerinage à Saint-Jacques de Compostelle; il s'agit d'une croix en or. Elle me raconte que cela la touche profondément dans sa foi. Elle croit fortement en Dieu et se confie à lui tous les jours. 


\section{Troisième rencontre : un partage}

La troisième rencontre débute par un partage sur ce qui s'est produit depuis notre dernière rencontre. Josée me raconte qu'elle pense se trouver un emploi à temps partiel. Je profite alors de l'occasion pour normaliser sa décision dans le contexte de son estime de soi et de l'avantage de pouvoir développer ses habiletés sociales. La suite de l'entrevue se passe dans un partage puisque j'ai apporté un disque compact de musique grégorienne. Nous écoutons alors deux plages de cette musique et j'en profite pour lui demander comment elle se sent lorsqu'elle écoute ce genre de musique.Je partage ensuite avec elle ce que j'aime de cette musique. Comme elle m'avait partagé le sens de deux mémentos, j'ai partagé ensuite un poème sur les choix que nous avons à faire dans la vie. Le poème est de Robert Frost et s'intitule The Road not Taken (p.131). La qualité de ce texte se trouve dans le message qui nous amène à réfléchir sur les choix que nous faisons dans la vie. Que nous prenions le chemin à gauche ou à droite, ce qui importe c'est de faire un choix qui nous amènera quelque part dans la vie.

\section{Quatrième rencontre : un approfondissement de ce qu'elle veut atteindre et changer}

À cette rencontre, Josée arrive sans ses lunettes fumées noires et, pour la première fois, elle peut soutenir un regard direct avec moi. Elle parle plus aisément et semble prête à avancer et à se distancer davantage de sa dépression. Elle me raconte comment elle veut se trouver un emploi à temps partiel et comment elle aimerait interagir davantage avec les gens.

Ce qui est intéressant de souligner ici, c'est l'évolution du rôle du thérapeute dans l'espace temporel. Ce rôle évolue en passant à travers différents stages; il commence comme un 
intervenant tout puissant au début, mais il indique que l'individu doit prendre la position d'autonomie. Cette attitude enlève toutes pensées que l'intervention est magique. Dans la phase initiale du processus, l'intervenant avait comme fonction d'activer le stress, de développer avec la cliente un plus grand éventail de buts possibles, et d'agir comme agent stimulateur de ce que voulait la cliente. Dans la phase finale, l'intervenant observe et reconnait que la cliente doit maintenant initier la marche vers l'atteinte de ses buts.

\section{Cinquième rencontre : un progrès}

Ce matin, Josée arrive toute rayonnante. Elle se sent très fière de m'annoncer que finalement elle a un emploi à temps partiel dans un dépanneur et qu'elle aime interagir avec les clients, même si ces conversations sont courtes.

La cliente devient davantage en contrôle de sa vie en réalisant un premier choix qui l'amènera vers un mieux être intérieur.

\section{Sixième rencontre : terminaison et support}

Finalement, comme nous approchons du temps des vacances d'été, il devient de plus en plus clair que Josée, pour l'instant, préférerait pouvoir jouir de son temps pour pratiquer ses sports préférés le tennis et la natation - et qu'elle aime son nouveau travail à temps partiel. Nous avons donc convenu qu'elle pouvait me contacter à l'automne si elle en ressentait le besoin. Nous nous sommes quittés sur ces dimensions positives.

Selon Whitaker, la cliente est la personne qui sait le mieux quand le processus d'aide doit se terminer. Quand elle indique clairement que le cheminement l'a amenée à une telle décision, il est temps de reconnaître que c'est elle qui a le pouvoir de mettre fin à nos rencontres. 


\section{Bibliographie}

FROST, Robert (1963), Complete Poems of Robert Frost, New York, Holt, Rinehart and Winston.

NEILL, John R. et David P. KNISKERN (Eds.) (1982), From Psyche to System. The Evolving Therapy of Carl Whitaker, New York, Guilford Press.

RYAN, Margaret O. (Ed.) (1989), Midnight Musings of a Family Therapist, New York, W.W.Norton.

WHITAKER, Carl A. et William M. BUMBERRY (1988), Dancing with the Family. A Symbolic Experiential Approach, New York, Brunner/Mazel. 